\title{
O ESTRESSE, O PROFESSOR E O TRABALHO DOCENTE
}

\section{STRESS, TEACHER AND TEACHING WORK}

Geyse Chrystine Pereira Souza Fernandes ${ }^{1}$

Luc Vandenbergue ${ }^{2}$

\section{RESUMO}

As condições estressantes enfrentadas na rotina diária do professor podem levar a um desequilíbrio entre o trabalho e a saúde física e mental, resultando no desenvolvimento do estresse, como a Síndrome de Burnout - SB. Diante disso, este estudo buscou, na literatura, quais as relações entre estresse e trabalho docente. O presente estudo trata-se de uma revisão do tipo integrativa. Os resultados apontaram vários assuntos em torno do estresse. Assim formaram-se duas categorias que abrangem A Síndrome de Bournout e Outros Transtornos. Os resultados apontam que o excesso de atividades e o desgaste emocional a que os docentes estão sujeitos no trabalho os tornam mais susceptíveis e vulneráveis ao desenvolvimento de transtornos relacionados ao estresse.

PALAVRAS-CHAVE: estresse; trabalho docente; professor.

\begin{abstract}
The stressful conditions faced in the teacher's daily routine can lead to an imbalance between work and physical and mental health, resulting in the development of stress, like Burnout Syndrome - SB. Therefore, this study sought, in the literature, the relations between stress and teaching work. The present study is about a review of the integrative type. The results pointed to several issues around stress. Thus two categories were formed covering Bournout Syndrome and Other Disorders. The results indicate that the excess of activities and the emotional exhaustion that the teachers are subject to at work make them more susceptible and vulnerable to the development of stress-related disorders.
\end{abstract}

KEY WORDS: stress - teacher work - teacher.

\section{INTRODUÇÃO}

As pesquisas sobre a saúde mental de professores evidenciam uma deterioração progressiva das condições de imagem e organização do trabalho dessa categoria (BATISTA et al, 2016). Nos últimos anos, as condições de trabalho estressantes para os professores têm se tornado cada vez mais um problema em muitos países. As condições estressantes enfrentadas na rotina diária do professor podem levar a um desequilíbrio entre o trabalho e a saúde física e mental, resultando no desenvolvimento do estresse (CEZAR-VAZ et al, 2015).

\footnotetext{
${ }^{1}$ Mestranda do Programa de Pós-Graduação em Ciências Ambientais e Saúde - PPGCAS da Pontifícia Universidade Católica de Goiás - PUC Goiás - Bolsista CAPES. Email: geysefisioterapia@gmail.com

${ }^{2}$ Doutor em Psicologia, Professor do Programa de Pós-Graduação em Ciências Ambientais e Saúde - PPGCAS da Pontifícia Universidade Católica de Goiás - PUC Goiás. Email: luc.m.vandenberghe@gmail.com
} 
Dentre os trabalhadores, os professores estão expostos a inúmeras fontes de pressão, além daquelas decorrentes das alterações no sistema trabalhista, como elevada carga horária de trabalho, pequenas pausas destinadas ao descanso, ritmo intenso de trabalho e exigências de um alto nível de atenção e concentração. Somam-se a esses uma elevação do nível de estresse levando à alterações na qualidade de vida dessa categoria consideravelmente comprometida ocasionando diversos distúrbios à saúde (LIMA JR e SILVA, 2014).

O excesso de atividades e o desgaste emocional a que os docentes estão sujeitos no trabalho tornam-os mais susceptíveis e vulneráveis ao desenvolvimento de transtornos relacionados ao estresse (DALAGASPERINA e MONTEIRO, 2014), tal como a Síndrome de Burnout - SB. A SB caracteriza-se como um fenômeno psicossocial que ocorre como uma resposta crônica aos estressores interpessoais ocorridos na situação de trabalho principalmente quando existe excessiva pressão, conflitos, poucas recompensas emocionais e de reconhecimento (BORBA et al, 2015).

O estresse profissional é também percebido como resultante das condições laborais e individuais, uma vez que as exigências impostas ao professor ultrapassam sua capacidade de lidar com elas (SILVEIRA, ENUMO e BATISTA, 2014).

A síndrome de Burnout se refere a uma experiência individual negativa, que decorre das relações interpessoais no ambiente de trabalho e tem como alvo os profissionais dos serviços humanos, como: médicos, professores, policiais. Composta, inicialmente, por três dimensões, a SB foi explicada pela exaustão emocional, pela despersonalização e pela diminuição da realização pessoal no trabalho (DALAGASPERINA e MONTEIRO, 2014). Apesar de apontar diferenças nos índices de Burnout, os estudos revelam a presença da Síndrome em todos os níveis de ensino, sendo, portanto, uma questão que abrange toda a categoria profissional.

A docência é considerada pela Organização Internacional do Trabalho (OIT) como uma atividade de risco desde 1981, uma vez que os professores compõem a segunda categoria profissional mais acometida por doenças ocupacionais em nível mundial (BORBA et al, 2015).

Dentre as doenças ocupacionais em professores, as relacionadas a fatores psíquicos estão crescendo em muitos países. Como causas de adoecimento encontram-se: ritmo intenso e precárias condições de trabalho, aumento da exigência cognitiva, perda de autonomia em sala de aula, falta de acompanhamento técnico, políticas de educação insuficientes, salários insatisfatórios, não reconhecimento social do trabalho, 
indisciplina/violência e desinteresse dos alunos. Nesse sentido, as condições e organização do trabalho do professor apresentam características que o expõem a fatores estressantes, os quais, se persistentes, podem levar à Síndrome de Burnout - SB (BORBA et al, 2015).

A literatura tem apontado como estressores do ambiente escolar: lidar com a classe e manter a disciplina; aplicar as tarefas; organizar grupos de trabalho; ajudar crianças com problemas comportamentais; preparar recursos para lições; lidar com incidentes envolvendo comportamento desafiador e indisciplina; falta de suporte diante de problemas comportamentais dos alunos; excesso de trabalho e falta de tempo; diferenças de desenvolvimento e motivação dos alunos e políticas ducacionais; a pressão exercida pelos pais. Esses estressores podem ocorrer nas escolas multisseriadas, onde os professores lidam com alunos de diferentes faixas etárias e séries ao mesmo tempo em uma mesma sala de aula (SILVEIRA, ENUMO e BATISTA, 2014).

Além da identificação dos estressores, é importante a análise dos mecanismos para sua regulação. A autorregulação do estresse está relacionada aos mecanismos de coping, ou enfrentamento. Sabe-se também que as estratégias de enfrentamento podem sofrer influências do gênero, do contexto no qual o indivíduo está inserido, das características pessoais e do trabalho (SILVEIRA, ENUMO e BATISTA, 2014).

A tensão derivada das condições adversas de trabalho pode desenvolver sintomas que vão desde as fases iniciais do estresse até a instalação da síndrome de burnout. A profissão docente é uma das categorias mais acometidas pela SB (DALAGASPERINA e MONTEIRO, 2014).

Assim o processo saúde-doença do docente pode estar ligado à sua atividade e às condições que a mesma é realizada considerando que a presença destes determinantes interferem no bem-estar do organismo e, consequentemente, na qualidade de vida dos professores (BAIÃO e CUNHA, 2013) podem causar disfunções e queda na performance profissional, acarretando estresse físico e emocional, a partir disso o trabalhador dispensará tempo e recursos financeiros para sua recuperação ou serão gastos recursos públicos para proporcionar tratamento e amparo financeiro a um trabalhador momentânea ou permanentemente improdutivo (MARINHO, 2012).

Nesse sentido, o foco da análise da problematização empreendida situa-se em identificar se o trabalho está ou não associado ao estresse em docentes. 


\section{METODOLOGIA}

O presente estudo trata-se de uma revisão do tipo integrativa que visa sintetizar as evidências disponíveis acerca do tema em questão. As etapas da elaboração da presente revisão integrativa foram as seguintes: estabelecimento da hipótese e objetivos da revisão integrativa; estabelecimento de critérios de inclusão e exclusão de artigos (seleção da amostra); definição das informações a serem extraídas dos artigos selecionados; análise dos resultados e discussão.

A questão norteadora desta revisão integrativa foi: $O$ trabalho docente causa estresse?

A busca de publicações indexadas ocorreu em Novembro/Dezembro de 2016 e para a seleção dos artigos foi utilizada a base de dados BVS. Os critérios de inclusão dos artigos definidos para a presente revisão integrativa foram: artigos de pesquisa publicados em português, inglês e espanhol com os textos completos disponíveis na base de dados selecionada, no período compreendido entre 2014 a 2016. Foram excluídos os artigos de revisão, os artigos que, apesar de trazer os critérios de inclusão, acima descritos, não atendiam ao objetivo do estudo e as intervenções.

As palavras-chave utilizadas foram: estresse AND trabalho AND docente. A busca foi realizada pelo acesso on-line e, utilizando os critérios de inclusão, a amostra final desta revisão integrativa foi constituída de quinze artigos, exposta no Quadro 1.

Após a leitura das pesquisas selecionadas na íntegra, prosseguiu-se com a análise e organização das temáticas: A Síndrome de Bournout e Outros Transtornos. Com o intuito de descrever e classificar os resultados, evidenciando o conhecimento produzido sobre o tema proposto, realizou-se a análise, categorização e síntese das temáticas. 
Quadro 1 - Artigos da amostra final, por ordem decrescente do ano de publicação.

\begin{tabular}{|c|c|c|c|c|}
\hline Autores & Título & $\begin{array}{l}\text { Periódico e ano de } \\
\text { publicação }\end{array}$ & Tipo de estudo & $\begin{array}{l}\text { Local da } \\
\text { pesquisa }\end{array}$ \\
\hline BATISTA et al & $\begin{array}{l}\text { Mental disorders in university teachers: } \\
\text { study in a service of medical investigation }\end{array}$ & $\begin{array}{l}\text { Journal of Research } \\
\text { Fundamental Care } \\
\text { online, } 2016\end{array}$ & $\begin{array}{c}\text { Retrospectivo, de } \\
\text { natureza documental }\end{array}$ & $\begin{array}{c}\text { Joao Pessoa, } \\
\text { Paraiba - Brasil }\end{array}$ \\
\hline $\begin{array}{l}\text { FERRACCIU et } \\
\text { al }\end{array}$ & $\begin{array}{l}\text { Índice de capacidade para o trabalho e } \\
\text { desequilíbrio esforço-recompensa } \\
\text { relacionado ao distúrbio de voz em } \\
\text { professoras da rede estadual de Alagoas }\end{array}$ & Rev. CEFAC., 2015 & $\begin{array}{l}\text { Epidemiológico de corte } \\
\text { seccional analítico }\end{array}$ & $\begin{array}{l}\text { Alagoas - } \\
\text { Brasil }\end{array}$ \\
\hline $\begin{array}{l}\text { CEZAR-VAZ } \\
\text { et al }\end{array}$ & $\begin{array}{c}\text { Mental Health of Elementary Schoolteachers } \\
\text { in Southern Brazil: Working Conditions and } \\
\text { Health Consequences }\end{array}$ & $\begin{array}{c}\text { The Scientific World } \\
\text { Journal, } 2015\end{array}$ & $\begin{array}{c}\text { Exploratório descritivo, } \\
\text { por meio de entrevista } \\
\text { estruturada com } \\
\text { questionário }\end{array}$ & Sul do Brasil \\
\hline $\begin{array}{l}\text { BANNAI, A. ; } \\
\text { UKAWA, S. ; } \\
\text { TAMAKOSHI, } \\
\text { A } \\
\end{array}$ & $\begin{array}{l}\text { Long working hours and psychological } \\
\text { distress among school teachers in Japan }\end{array}$ & $\begin{array}{l}\text { Journal of } \\
\text { Occupational } \\
\text { Health, } \\
2015 \\
\end{array}$ & Transversal & $\begin{array}{l}\text { Hokkaido, } \\
\text { Japão }\end{array}$ \\
\hline BORBA et al & $\begin{array}{l}\text { Síndrome de Burnout em professores: estudo } \\
\text { comparativo entre o ensino público e privado }\end{array}$ & $\begin{array}{c}\text { Psicologia } \\
\text { Argumento, } 2015\end{array}$ & $\begin{array}{l}\text { descritivo, comparativo e } \\
\text { transversal, de } \\
\text { abordagem quantitativa }\end{array}$ & $\begin{array}{c}\text { Porto Alegre e } \\
\text { Região } \\
\text { Metropolitana } \\
\text { do Estado do } \\
\text { Rio Grande do } \\
\text { Sul/Brasil } \\
\end{array}$ \\
\hline QI X et al & $\begin{array}{l}\text { Relationship between work strain, need for } \\
\text { recovery after work and cumulative cortisol } \\
\text { among kindergarten teachers }\end{array}$ & $\begin{array}{l}\text { International } \\
\text { Archives of } \\
\text { Occupational and } \\
\text { Environmental } \\
\text { Health, 2015 }\end{array}$ & Não informado & Nanjing, China \\
\hline $\begin{array}{l}\text { LINDFELT, IP } \\
\text { e BARNETT }\end{array}$ & $\begin{array}{l}\text { Survey of career satisfaction, lifestyle, and } \\
\text { stress levels among pharmacy school faculty }\end{array}$ & $\begin{array}{c}\text { American Journal of } \\
\text { Health-System } \\
\text { Pharmacy, 2015 }\end{array}$ & Não informado & EUA \\
\hline $\begin{array}{c}\text { ESPINOZA- } \\
\text { DÍAZ, TOUS- } \\
\text { PALLARĖS e } \\
\text { VIGIL-COLET }\end{array}$ & $\begin{array}{l}\text { Efecto del Clima Psicosocial del Grupo y de } \\
\text { la Personalidad en el Síndrome de Quemado } \\
\text { en el Trabajo de los docentes }\end{array}$ & $\begin{array}{l}\text { anales de psicología, } \\
2015\end{array}$ & Não informado & $\begin{array}{l}\text { Tarragona, } \\
\text { Espanha }\end{array}$ \\
\hline $\begin{array}{l}\text { SILVEIRA, K } \\
\text { A; ENUMO, S } \\
\text { R F; BATISTA, } \\
\text { E P }\end{array}$ & $\begin{array}{c}\text { Indicadores de estresse e estratégias de } \\
\text { enfrentamento em professores de ensino } \\
\text { multisseriado }\end{array}$ & $\begin{array}{c}\text { Revista } \\
\text { Quadrimestral da } \\
\text { Associação } \\
\text { Brasileira de } \\
\text { Psicologia Escolar e } \\
\text { Educacional, 2014 }\end{array}$ & descritivo e correlacional & $\begin{array}{l}\text { Interior do } \\
\text { Estado do } \\
\text { Espírito Santo }\end{array}$ \\
\hline $\begin{array}{l}\text { MARTINS, M } \\
\text { F D et al }\end{array}$ & $\begin{array}{c}\text { O trabalho das docentes da Educação Infantil } \\
\text { e o mal-estar docente: o impacto dos } \\
\text { aspectos psicossociais no adoecimento }\end{array}$ & $\begin{array}{c}\text { Cadernos de } \\
\text { Psicologia Social do } \\
\text { Trabalho, 2014 } \\
\end{array}$ & $\begin{array}{c}\text { estudo de caráter } \\
\text { censitário e corte } \\
\text { transversal } \\
\end{array}$ & $\begin{array}{l}\text { Pelotas (RS), } \\
\text { Brasil }\end{array}$ \\
\hline $\begin{array}{l}\text { TIJDINK, J K } \\
\text { VERGOUWEN } \\
\text { A CM e } \\
\text { SMULDERS, Y } \\
\text { M }\end{array}$ & $\begin{array}{l}\text { Emotional exhaustion and burnout among } \\
\text { medical teachers. A national survey }\end{array}$ & $\begin{array}{c}\text { BMC Medical } \\
\text { Education, } 2014\end{array}$ & $\begin{array}{l}\text { Quantitativo com } \\
\text { questionário }\end{array}$ & Holanda \\
\hline $\begin{array}{l}\text { DALAGASPER } \\
\text { INA, P. \& } \\
\text { MONTEIRO, J. } \\
\text { K. }\end{array}$ & $\begin{array}{l}\text { Preditores da síndrome de burnout em } \\
\text { docentes do ensino privado }\end{array}$ & Psico-USF, 2014 & $\begin{array}{l}\text { Quantitativo de caráter } \\
\text { correlacional e } \\
\text { explicativo com } \\
\text { análises descritivas e de } \\
\text { regressão linear múltipla }\end{array}$ & $\begin{array}{l}\text { Rio Grande do } \\
\text { Sul, Brasil }\end{array}$ \\
\hline $\begin{array}{l}\text { BRAUM, A C e } \\
\text { CARLOTTO, } \\
\text { M S }\end{array}$ & $\begin{array}{c}\text { Síndrome de Burnout: estudo comparativo } \\
\text { entre professores do Ensino Especial e do } \\
\text { Ensino Regular }\end{array}$ & $\begin{array}{c}\text { Revista } \\
\text { Quadrimestral da } \\
\text { Associação } \\
\text { Brasileira de } \\
\text { Psicologia Escolar e } \\
\text { Educacional, 2014 }\end{array}$ & Não informado & $\begin{array}{l}\text { Vale do Rio dos } \\
\text { Sinos e Porto } \\
\text { Alegre e região } \\
\text { metropolitana }\end{array}$ \\
\hline $\begin{array}{c}\text { SÁNCHEZ- } \\
\text { OLIVA, D et al. }\end{array}$ & $\begin{array}{l}\text { Motivación y burnout en profesores de } \\
\text { educación física: incidencia de la frustración } \\
\text { de las necesidades psicológicas básicas }\end{array}$ & $\begin{array}{l}\text { Cuadernos de } \\
\text { Psicología del } \\
\text { Deporte, } 2014 \\
\end{array}$ & $\begin{array}{l}\text { Descritivo. Análise de } \\
\text { regressão linear }\end{array}$ & Espanha \\
\hline $\begin{array}{l}\text { MAHECHA } \\
\text { ANGULO, M; } \\
\text { LEÓN } \\
\text { ESPINOSA, E }\end{array}$ & $\begin{array}{c}\text { Condiciones salud-trabajo de docentes } \\
\text { profesionales de enfermería vinculados a } \\
\text { programas de formación para auxiliares de } \\
\text { enfermeira }\end{array}$ & $\begin{array}{l}\text { Enfermería Global } \\
\text { No }^{\circ} 35 \text { Julio } 2014\end{array}$ & $\begin{array}{l}\text { Descritivo de corte } \\
\text { transversal }\end{array}$ & $\begin{array}{l}\text { Tunja, } \\
\text { Colômbia }\end{array}$ \\
\hline
\end{tabular}




\section{RESULTADOS}

Nesta revisão foram encontrados 65 artigos que após o descarte dos repetidos, das revisões, dos indisponíveis, ou dos que não atendem ao objetivo a amostra final foi constituída de 15 artigos, conforme Quadro 1. Destes, 8 foram realizados no Brasil. Além de todos os artigos se tratarem de estresse, os mesmos variaram em seus focos de estudo se relacionando com os transtornos mentais de forma geral e também com a Síndrome de Burnout. Esta, por sua vez, pesquisada em 6 artigos.

Todos os estudos foram realizados com professores com prevalência do sexo feminino, porém em variados níveis e modalidades de ensino, incluindo assim o professor de ensino superior, ensino médio, ensino fundamental e ensino infantil variando entre público e privado, regular e especial e ainda multisseriada. Quanto ao tipo de pesquisa houve variados estudos e métodos dentro da pesquisa qualitativa e quantitativa, este em sua maioria. Tais como estudo transversal, retrospectivo e descritivo, prevalecendo em sua maioria. No que se refere aos periódicos dos artigos selecionados, foram identificados 14, com destaque para a Revista Quadrimestral da Associação Brasileira de Psicologia Escolar e Educacional, que publicou dois dos artigos.

\section{DISCUSSÃO: A SÍNDROME DE BURNOUT E OUTROS TRANSTORNOS}

A Síndrome de Burnout se refere a uma experiência individual negativa, que decorre das relações interpessoais no ambiente de trabalho e tem como alvo os profissionais dos serviços humanos, como: médicos, professores, policiais. Composta, inicialmente, por três dimensões, a SB foi explicada pela exaustão emocional, pela despersonalização e pela diminuição da realização pessoal no trabalho. Esse modelo foi ampliado, em 2005, por GilMonte, para quatro dimensões sendo estas, a ilusão pelo trabalho, o desgaste psíquico, a indolência e o sentimento de culpa (DALAGASPERINA e MONTEIRO, 2014). Apesar de apontar diferenças nos índices de Burnout, os estudos revelam a presença da Síndrome em todos os níveis de ensino, sendo, portanto, uma questão que abrange toda a categoria profissional.

A tensão derivada das condições adversas de trabalho pode desenvolver sintomas que vão desde as fases iniciais do estresse até a instalação da síndrome de Burnout. A profissão docente é uma das categorias mais acometidas pela SB (DALAGASPERINA \& MONTEIRO, 2014). 
No estudo que compara a prevalência de Burnout entre o ensino privado e público a prevalência da síndrome foi de $41,5 \%$ entre os professores das escolas públicas e 26,6\% entre os da rede privada. Contudo, não houve diferença estatisticamente significativa entre os grupos. Concluiu-se que tanto o contexto público de ensino quanto o privado apresentam estressores que podem levar os professores ao adoecimento, dado que permite refletir que a prevalência da Síndrome de Burnout pode estar mais relacionada à categoria profissional em si do que ao setor de trabalho (BORBA et al, 2015). Na pesquisa que identifica preditores de Bunout em docentes do ensino privado resultou que a maioria dos fatores preditores refere-se à organização do trabalho, ressaltando-se as dificuldades em relação aos alunos, tais como: falta de reconhecimento, falta de limite e de educação, dificuldades de relacionamento (DALAGASPERINA e MONTEIRO, 2014).

Os resultados de outro estudo, com professores do ensino infantil, primário e secundário, mostraram que tanto o clima psicossocial (particularmente o grau de desorganização) quanto a personalidade (particularmente a estabilidade emocional) influenciam o Burnout e, embora os fatores climáticos pareçam ser melhores preditores, o uso de medidas de personalidade aumenta a capacidade preditiva da síndrome (ESPINOZADÍAZ, TOUS-PALLARÈS e VIGIL-COLET, 2015). Já no estudo em professores de medicina da Holanda a exaustão emocional, ou Bunout, é comum entre eles, e são determinados por vários fatores, todos relacionados com estar em uma fase inicial de sua carreira profissional (TIJDINK, VERGOUWEN e SMULDERS, 2014).

Num estudo com professores de educação física de ensino público e privado da Espanha onde foi avaliada a importância das necessidades psicológicas básicas para explicar e predizer o tipo de motivação e os níveis de Burnout indicou como a frustração da necessidade de competência previa positivamente a desmotivação, exaustão emocional e o cinismo e negativamente a motivação intrínseca e a eficácia profissional; a frustração de autonomia previa positivamente a exaustão emocional e negativamente a eficácia profissional; e a frustração das relações sociais, previa positivamente, a eficácia profissional.

A divergência ficou por conta de um estudo que compara professores do ensino especial e regular onde os resultados não confirmam a hipótese de que professores do Ensino Especial possuem índices mais elevados de Burnout. Ao autores sugerem que pode-se entender este resultado a partir de questões culturais de que estresse e Burnout podem ter diferentes configurações, a depender do contexto cultural, social e político da população em 
estudo. Também podem ter influenciado a configuração do trabalho, o numero menor de alunos e a capacitação continuada específica (BRAUM e CARLOTTO, 2014).

No que se refere aos problemas relacionados às condições de trabalho, há consenso na literatura quanto aos diversos problemas enfrentados pelos professores. Os autores referem estudos nos quais são destacados aspectos como: precárias condições de trabalho (iluminação, espaço físico, material didático); organização do trabalho (multiplicidade de tarefas diferenciadas, ritmos de trabalho, aumento das exigências cognitivas, perda de autonomia, burocratização, rotinização das atividades educativas); aspectos físicos (posturas desconfortáveis, uso elevado e frequente da voz, peso de grandes quantidades de materiais); aspectos relacionais (falta de diálogo com a administração e equipe técnica, dificuldades nas relações com as famílias dos alunos, falta de valorização do trabalho realizado, estado psicológico, disciplina de alunos); e aspectos sociais (baixos salários, trabalho em mais de uma escola, imagem errônea da opinião pública sobre o professor) (BRAUM e CARLOTTO, 2014).

Num estudo sobre professores universitários a depressão foi responsável por 53\% dos afastamentos de professores, a esquizofrenia (12\%), o transtorno bipolar (10\%), a reação aguda ao estresse (8\%), a ansiedade (7\%), os transtornos delirantes (4\%) e outros (8\%). Concluindo assim que o transtorno mental que mais acomete a categoria docente pesquisada é a depressão (BATISTA et al, 2016).

Professoras que possuem mais de 11 anos de docência, lecionam em duas ou mais escolas e trabalham em escolas que sempre têm depredações e violência contra os funcionários apresentam maiores chances de ter distúrbio de voz. Isso foi identificado na pesquisa que relaciona o distúrbio de voz com o estresse psicossocial. Foi observado que o estresse psicossocial no trabalho não mostrou significância com a presença do distúrbio de voz, mas teve associação com a faixa etária em que se observa alto DER (Escala Desequilíbrio Esforço-Recompensa) nas professoras mais jovens (FERRACCIU et al, 2015).

As cinco condições de trabalho estressantes listadas pelos professores do ensino fundamental incluem salário inadequado $(56,8 \%)$, material de trabalho insuficiente ou inadequado $(54,1 \%)$, tamanho da sala de aula inadequado para o número de alunos $(50 \%)$, atividades excessivas $(35,1 \%)$, e levar o trabalho para casa $(32,4 \%)$. As cinco conseqüências biopsicossociais mais freqüentes foram as condições de trabalho, incluindo ansiedade $(78,4 \%)$, cefaléia $(59,5 \%)$, estresse $(54,1 \%)$, acordar várias vezes durante a noite $(51,4 \%)$ e irritabilidade $(48,6 \%)$. Os professores indicaram que as relações de trabalho que promovem o 
relacionamento $(73 \%)$, o respeito $(67,6 \%)$ e a harmonia $(59,5 \%)$ favorecem o bem-estar (CEZAR-VAZ et al, 2015).

A angústia psicológica foi identificada em 47,8\% dos homens e 57,8\% nas mulheres, em professores do ensino secundário de Hokkaido, Japão. Os resultados mostraram um risco significantemente aumentado apenas em homens que trabalham> 60 horas por semana em comparação com aqueles que trabalham $\leq 40$ horas por semana. Não houve associações significativas entre longas horas de trabalho e sofrimento psicológico para as mulheres (BANNAI, UKAWA e TAMAKOSHI, 2015).

No ensino multisseriado da zona rural identificou-se, em 57\% da amostra, estresse relacionado à ansiedade (moderada a grave em 30\%). Os estressores mais frequentes foram pouco acompanhamento familiar e problemas motivacionais e comportamentais dos alunos (SILVEIRA, ENUMO e BATISTA, 2014).

No ensino infantil resultados revelaram que um grupo significativo de professoras está em risco de adoecimento, trabalhando em um ambiente que exige um alto grau de envolvimento, possuindo pouco controle sobre esse trabalho. A interação entre o trabalho e o ambiente laboral, a satisfação no trabalho e as condições de organização em que essas professoras exercem seus ofícios, da forma como estão, podem levá-las ao mal-estar docente (MARTINS, et al, 2014).

Um estudo usou o fator químico Cortisol em professores do jardim de infância e não foram encontradas correlações significativas entre as demandas de trabalho ou controle de trabalho e HCC (concentração de cortisol do cabelo). NFR (necessidade subjetiva de recuperação) foi significativamente e inversamente correlacionado com $\mathrm{HCC}(\mathrm{r}=-0,41, \mathrm{p}=$ 0,006). A associação inversa entre NFR e HCC permaneceu significativa quando a idade e as demandas de trabalho e o controle de trabalho foram controlados para $(\mathrm{p}=0,02)$. Concluindo que a atividade do eixo hipotálamo-hipófise-adrenal declina com a acumulação de NFR após o tempo de trabalho (QI et al, 2015).

A pesquisa com professores de uma faculdade de farmácia dos EUA revelou altos níveis de satisfação no trabalho entre os respondentes, mas foram encontrados níveis mais baixos de satisfação com o equilíbrio entre a vida profissional e familiar e níveis comparáveis de estresse para a população em geral (LINDFELT e BARNETT, 2015).

No artigo que descreve as condições de saúde e de trabalho dos docentes das instituições de formação das enfermeiras vinculadas aos auxiliares de enfermagem, permitindo visibilizar as características sócio-demográficas dessa população, sua saúde e 
condições de trabalho dentro e fora do trabalho resultou que a saúde e o trabalho mais favoráveis foram encontrados em professores que trabalham nos dois ciclos (teoria e prática) e tem um contrato com todos os benefícios e afiliação de ala de segurança social abrangente. $\mathrm{O}$ pior naqueles que trabalham somente na prática ou na teoria, aqueles que relataram mais sintomas de distúrbios físicos, emocionais e comportamentais, mas também direitos laborais menos reconhecidos (MAHECHA ÂNGULO e LEÓN ESPINOSA, 2014).

Os problemas de saúde que afetam a categoria estão relacionados a um conjunto de fatores: o tipo de trabalho exercido, tendo em vista a responsabilidade pela formação de outros sujeitos; o excesso de trabalho; a precarização do trabalho, à perda de autonomia, à sobrecarga de trabalho burocrático, ao quadro social e econômico e às condições de vida dos alunos (VIEIRA, 2014). Os professores preparam e ministram aulas, corrigem provas e trabalhos e precisam manter-se constantemente atualizados nos conteúdos que transmitem. Além destas tarefas rotineiras, também se ocupam da gestão escolar, que inclui atividades de planejamento, elaboração de projetos, avaliação de currículos, serviços administrativos, orientação de alunos, atendimento aos pais, envolvimento na elaboração de atividades extraescolares e participação de reuniões e assembléias. Alem disso convivem com a desvalorização do trabalho (DALAGASPERINA \& MONTEIRO, 2014).

\section{CONSIDERAÇÕES FINAIS}

Os resultados deste estudo mostram que as principais condições de trabalho estressantes para os professores estão relacionados com as consequências biopsicossociais para a saúde dos professores. Além da identificação dos estressores, é importante a análise dos mecanismos de enfrentamento que podem sofrer influências do gênero, do contexto no qual o indivíduo está inserido, das características pessoais e do trabalho.

$\mathrm{O}$ excesso de atividades e o desgaste emocional a que os docentes estão sujeitos no trabalho tornam-os mais susceptíveis e vulneráveis ao desenvolvimento de transtornos relacionados ao estresse.

\section{REFERÊNCIAS}

BAIÃO, L. P. M.; CUNHA, R. G. Doenças e/ou disfunções ocupacionais no meio docente: uma revisão de literatura. Revista Formação@Docente. v. 5, n. 1, p. 6-21, 2013.

BANNAI, A. ; UKAWA, S. ; TAMAKOSHI, A. Long working hours and psychological distress among school teachers in Japan. J Occup Health 2015; 57: 20-27 
BATISTA et al. Mental disorders in university teachers: study in a service of medical investigation. J. res.: fundam. care. online 2016. abr./jun. 8(2):4538-4548.

BRAUM, A C; CARLOTTO, M S. Síndrome de Burnout: estudo comparativo entre professores do Ensino Especial e do Ensino Regular. Revista Quadrimestral da Associação Brasileira de Psicologia Escolar e Educacional , SP. Volume 18, Número 1, Janeiro/Abril de 2014: 125-133.

BORBA et al. Síndrome de Burnout em professores: estudo comparativo entre o ensino público e privado. Psicol Argum. 2015 jan./abr., 33(80), 270,281

CEZAR-VAZ et al. Mental Health of Elementary Schoolteachers in Southern Brazil: Working Conditions and Health Consequences. The Scientific World Journal, 2015

DALAGASPERINA, P.; MONTEIRO, J. K. Preditores da síndrome de burnout em docentes do ensino privado. Psico-USF, Bragança Paulista, v. 19, n. 2, p. 265-275, maio/agosto 2014

ESPINOZA-DÍAZ, I M; TOUS-PALLARÈS, J; VIGIL-COLET, A. Efecto del Clima Psicosocial del Grupo y de la Personalidad en el Síndrome de Quemado en el Trabajo de los docentes. anales de psicología, 2015, vol. 31, nº 2 (mayo), 651-657.

FERRACCIU et al. Índice de capacidade para o trabalho e desequilíbrio esforço-recompensa relacionado ao distúrbio de voz em professoras da rede estadual de Alagoas. Rev. CEFAC. 2015 Set-Out; 17(5):1580-1589.

LIMA JÚNIOR J. P.; SILVA, T.F. Análise da sintomatologia de distúrbios osteomusculares em docentes da Universidade de Pernambuco - Campus Petrolina. Rev Dor. São Paulo, outdez;15(4):276-80, 2014.

LINDFELT, T A; IP, E J; BARNETT, M J. Survey of career satisfaction, lifestyle, and stress levels among pharmacy school faculty. Am J Health-Syst Pharm-Vol 72 Sep 15, 2015.

MAHECHA ANGULO, M; LEÓN ESPINOSA, E. Condiciones salud-trabajo de docentes profesionales de enfermería vinculados a programas de formación para auxiliares de enfermería. Enfermería Global. № 35 Julio, 2014.

MARINHO, E. F. Estudo das principais queixas álgicas indicativas de DORT em professores de educação especial. Pós-graduação em Ortopedia e Traumatologia com ênfase em Terapia Manual - Faculdade Ávila, 2012.

MARTINS, M F D et al. O trabalho das docentes da Educação Infantil e o mal-estar docente: o impacto dos aspectos psicossociais no adoecimento. Cadernos de Psicologia Social do Trabalho, 2014, vol. 17, n. 2, p.281-289 - DOI: 10.11606/issn.1981-.0490.v17n2p281-289.

QI X et al. Relationship between work strain, need for recovery after work and cumulative cortisol among kindergarten teachers. International Archives of Occupational and Environmental Health. February 2015 
SÁNCHEZ-OLIVA, D et al. Motivación y burnout en profesores de educación física: incidencia de la frustración de las necesidades psicológicas básicas. Cuadernos de Psicología del Deporte, vol. 14, 3, 75-82, 2014

SILVEIRA, K A; ENUMO, S R F; BATISTA, E P. Indicadores de estresse e estratégias de enfrentamento em professores de ensino multisseriado. Revista Quadrimestral da Associação Brasileira de Psicologia Escolar e Educacional , SP. Volume 18, Número 3, Setembro/Dezembro de 2014: 457-465.

TIJDINK, J K VERGOUWEN, A CM e SMULDERS, Y M. Emotional exhaustion and burnout among medical teachers. A national survey. BMC Medical Education, 2014.

VIEIRA, S R S. Sofrimento psíquico e trabalho. Rev. Latinoam. Psicopat. Fund., São Paulo, 17(1), 114-124, mar. 2014 